\title{
Bacterial flagellins: mediators of pathogenicity and host immune responses in mucosa
}

\author{
Hugo Cruz Ramos ${ }^{1}$, Martin Rumbo ${ }^{2}$ and Jean-Claude Sirard ${ }^{1}$ \\ ${ }^{1}$ Equipe AVENIR-INSERM d'Immunité Anti-microbienne des Muqueuses, E0364, Institut Pasteur de Lille, Institut de Biologie, \\ 1 rue du Professeur Calmette, BP 447 - 59021 Lille, France \\ ${ }^{2}$ Catedra de Inmunologia, Facultad de Ciencias Exactas, Universidad Nacional de La Plata, 47 y 115 (1900) La Plata, Argentina
}

\begin{abstract}
Flagella contribute to the virulence of pathogenic bacteria through chemotaxis, adhesion to and invasion of host surfaces. Flagellin is the structural protein that forms the major portion of flagellar filaments. Thus, flagellin consists of a conserved domain that is widespread in bacterial species and is dedicated to filament polymerization. Conversely, mammalian hosts detect the conserved domain on flagellin monomers through Toll-like receptor (TLR) 5, which triggers proinflammatory and adaptive immune responses. This review describes the relationships among flagellin molecular structure, bacterial virulence and host defenses, with special emphasis on mucosal tissues.
\end{abstract}

Motile bacteria display complex surface organelles, known as flagella, which are up to $15 \mu \mathrm{m}$ long. Flagellar activity is coupled to chemotaxis machinery that senses environmental chemical and physical information and orchestrates migration for bacterial growth and survival [1]. Three parts are distinguished in the flagellar structure: a basal body that functions as a motor dependent on proton motive force, a torsion hook and a helical hollow filament (referred to as the flagellum) [1]. Bacterial motility alternates between a run (lasting a few seconds) and a tumble (lasting a fraction of second). During a run, the motor rotates the left-handed helical flagellar filament counterclockwise, which forms a bundle and propels the cell. A tumble is caused by quick reversal of the motor rotation, which switches to a right-handed filament. Here, we summarize the structural and functional organization of the flagellum of the enteropathogenic bacteria Salmonella enterica serovar Typhimurium (Figure 1). The flagellar filament is composed of approximately 20000 subunits of a unique protein, known as flagellin (FliC, 495 amino acids; Genbank accession number AAL20871). Flagellin is secreted through the central channel of the growing filament to be assembled in a helical structure at the distal end $[1,2]$. A capping structure promotes polymerization at the tip and prevents release of subunits in the bacterial environment. Depending on the bacterial species, flagellins have molecular masses ranging from 28 to $80 \mathrm{kDa}$ [3]. Sequence alignments show the conserved termini regions

Corresponding author: Jean-Claude Sirard (jean-claude.sirard@ibl.fr).

Available online 22 September 2004
( $\sim 170 \mathrm{~N}$ - and $100 \mathrm{C}$-terminal residues) flanking a central region, which is hypervariable both in residue composition and size. The $\mathrm{N}$ - and $\mathrm{C}$-terminal chains of flagellin form packed $\alpha$-helix structures, which constitute D0 and D1 domains, positioned in the filament core [2] (Figure 1). The variable region of flagellin is exposed as a $\beta$-sheet folded structure (D2 and D3 domains) on the filament outer surface. Monomers of flagellin in solution are compactly folded, except for the most terminal regions, which are disordered [4].

Flagella are essential structures for the pathogenic potential of bacteria because they provide motility or increase adhesion. By contrast, recent findings highlight a major role of the flagellin monomer in the detection of microbes by the host and in the induction of immune responses.

\section{Contribution of flagella to pathogenicity}

The prerequisite event for any infection is the encounter of pathogenic bacteria with the target tissue. Plants, insects and mammals are all dealing with flagellated pathogens. Mucosal surfaces, especially epithelia, are the main sites of mammal host-pathogen interactions. Pathogenic bacteria specifically produce flagella to promote colonization and invasion of mucosa [5-7]. In mucosa, the flagellar structure is required for motility, adhesion, invasion and secretion of virulence factors.

\section{Motility and pathogenesis}

The glycocalyx and mucus layer associated with epithelium form inevitable physical and chemical obstacles for pathogens. Similarly, dynamic processes, such as the upward flow of mucus from the bronchial epithelia or peristalsis in the intestine, have to be counterbalanced by pathogens if colonization is to be achieved. In the host, motility combined with chemotaxis enables fine-tuned access of pathogens to the target mucosal tissues (Figure 2). Motility functions of Helicobacter pylori and Pseudomonas aeruginosa are crucial for infection of the stomach and lungs, respectively [8,9]. Colonization of intestinal mucosa by Vibrio cholerae strictly requires motility [7]. Colonization of rabbit appendix by $S$. enterica serovar Typhimurium also depends on accessibility and motility [10]. Because motility increases the occurrence of host-pathogen 

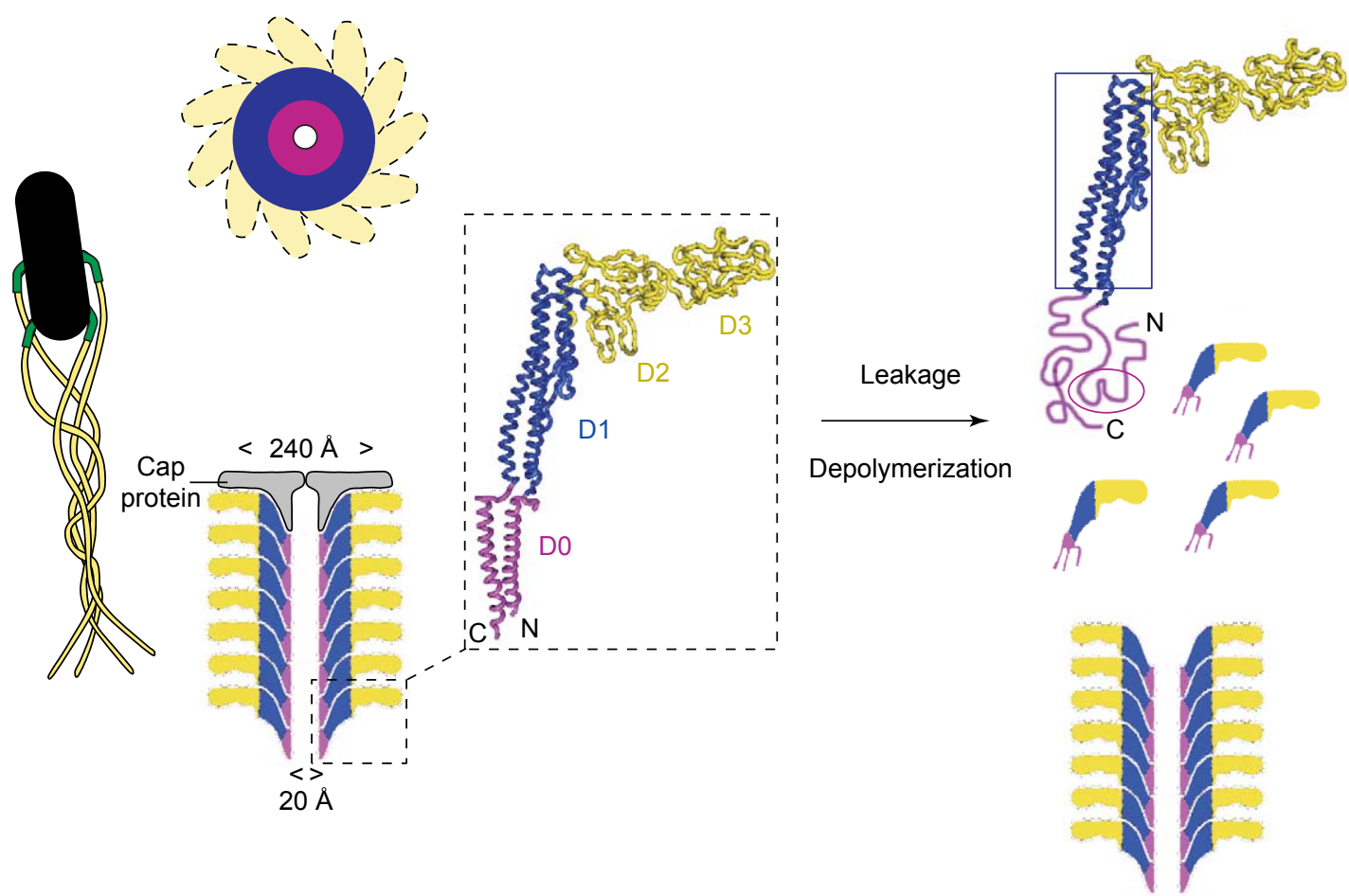

$\overline{\text { TRENDS in Microbiology }}$

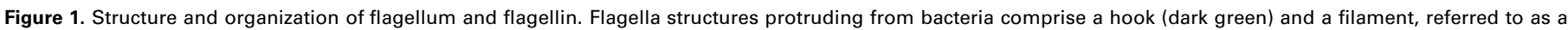

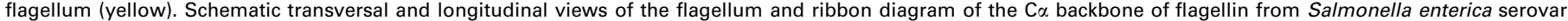

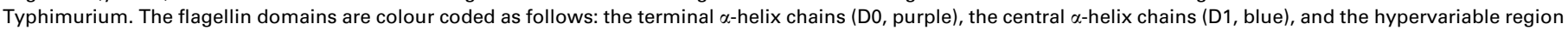

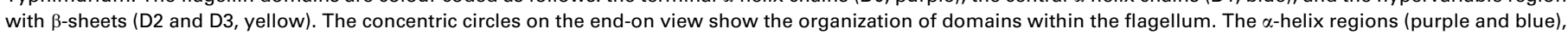

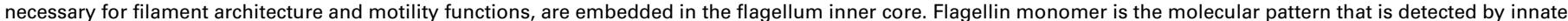

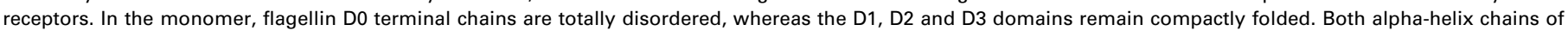

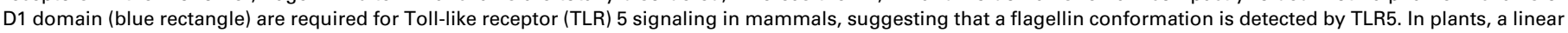
motif in the $\mathrm{N}$-terminus (purple circle) is required for defenses mediated by flagellin-sensitive receptor (FLS2).

interactions, this feature contributes to the main role of the flagellum in pathogenesis.

\section{Role of flagella in adhesion to and invasion of mucosal surfaces}

Flagella can participate in the occupancy of a specific niche by acting as an adhesin (Figure 2). Crude flagella from the opportunistic pathogen Clostridium difficile bind to cecal mucus of germ-free mice [11]. In addition, nonflagellated $C$. difficile associate with the cecal tissue at a rate that is 10-fold lower than that of a flagellated strain, highlighting the in vivo role of flagella in adherence to mucus. In cystic fibrosis, $P$. aeruginosa colonizes the airway lumen at a distance several micrometers away from the surface. Noticeably, $P$. aeruginosa flagellin binds mucin (Muc1), an abundant component of airway mucus [12]. Furthermore, enteropathogenic Escherichia coli adhere to the intestinal mucosa or to tissue culture cells using a flagellum-dependent mechanism [13]. The hypervariable region of flagellin (D2-D3) probably bears the adhesin-like properties (Figures 1 and 2). The importance of flagella for the invasion of epithelial cells has been reported for several bacteria, including Yersinia enterocolitica [5,14].

\section{Flagellum as a secretion system for toxins}

The machinery for flagellum biosynthesis is the paradigm of type III secretion systems (TTSSs) [1]. In pathogenic bacteria, TTSSs serve as molecular syringes that are required for export and injection of virulence factors into the cytosol of host cells [15]. Consequently, pathogens hijack cytosolic pathways to colonize or kill the host cells. The flagellar TTSS can be an additional mechanism for the export of virulence factors (Figure 2). In Y. enterocolitica, flagellum mediates secretion of several extracellular toxins, including the phospholipase YplA [16].

\section{Coordinated expression of virulence genes and genes involved in flagellum synthesis}

The transcription of $\sim 50$ flagellar genes is hierarchically controlled by environmental conditions via the master regulator operon flhDC [17]. In Salmonella, flagellar genes and loci encoding virulence factors are part of regulons that are dependent on FlhD-FlhC and two-component sensor kinase and response regulators, such as $\mathrm{PhoP}-\mathrm{PhoQ}$ or BarA-SirA [17,18]. In V. cholerae, the ToxR regulatory system coordinates the transcription of motility genes and specific virulence genes in response to environmental conditions [7]. By contrast, the BvgAS system of Bordetella bronchiseptica represses flagellum gene transcription and activates the expression of virulence factors [19]. In general, expression of flagella is probably switched off once mucosal pathogenic bacteria disseminate into deeper tissues [6]. 


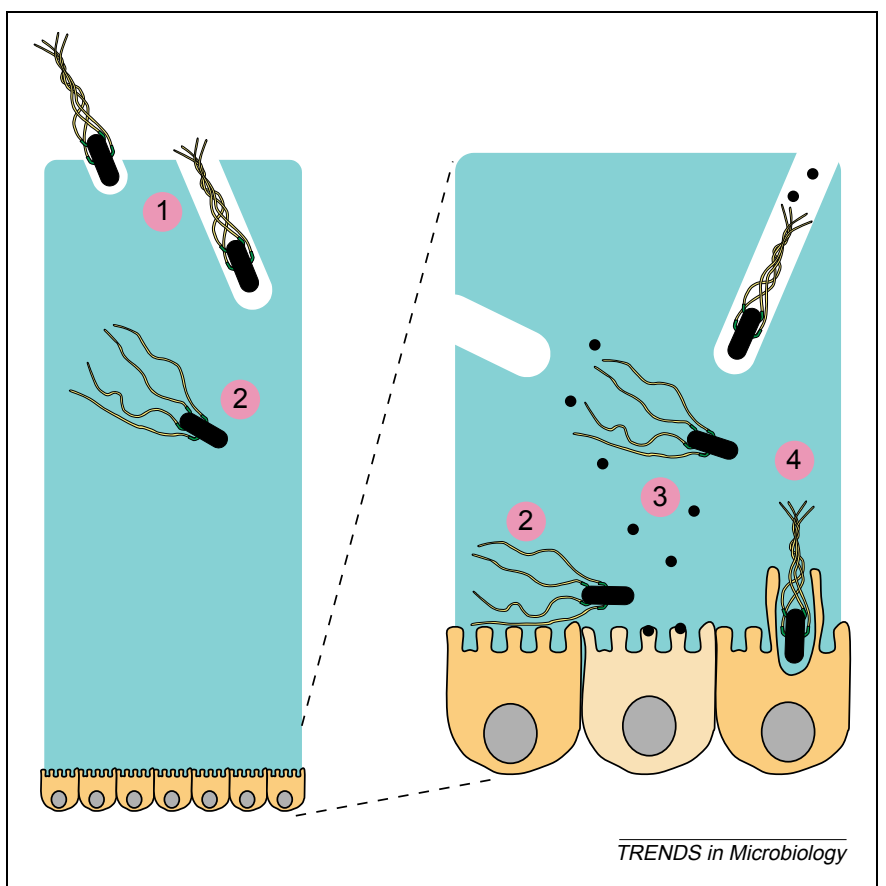

Figure 2. Contribution of flagellum to bacterial pathogenesis in mucosa. (1) Motility combined with chemotaxis participate in the penetration and colonization of specific niches in mucosa. (2) Bacterial adhesion to mucus or to the epithelial cell surface is assisted by flagella. The outer hypervariable domain of flagellin is probably involved in this function, suggesting a dependence on serotype. (3) Upon bacterial penetration of mucus, virulence factors (black dots) can be specifically exported by the flagellar secretion system to poison the host or hijack machinery of epithelial cells (light-colored). (4) Flagella promote bacterial invasion during infection.

\section{Mucosal proinflammatory responses to flagellin}

Colonization of mucosa is restricted by the renewal of epithelial cells, the barrier function of the intercellular junctions, and the production of antimicrobial molecules (i.e. lysozyme and defensins), which clear microbes [20]. Because pathogens are equipped to breach the mucosal barriers, hosts need to respond rapidly to circumvent dissemination of bacteria. These transient defenses, classified as a proinflammatory response or an inducible innate immune response, are stimulated by mucosal sentinel cells. Although macrophages, monocytes and dendritic cells (DCs) are the main sentinels in the systemic compartment, epithelial cells represent the first and major cell type encountered by microorganisms in mucosa [20]. The sensing of pathogens is achieved by pattern-recognition receptors (PRRs) that detect conserved microbe-associated molecular patterns (MAMPs) [21]. The Toll-like receptor (TLR) family plays a key role in intra- and extracellular detection of MAMPs, such as bacterial lipopolysaccharide (LPS; TLR4), lipopeptides (TLR2), peptidoglycan (TLR2-TLR6), or unmethylated CpG DNA (TLR9) [21]. TLRs are transmembrane proteins that function as homo- or hetero-dimers [21]. They are organized into three functional domains: an extracellular leucine-rich repeat (LRR) module involved in MAMP recognition, a membrane-spanning motif and a Tollinterleukin (IL)-1 receptor domain (TIR) required for transmission of stimulus to adaptor molecules, such as MyD88 (myeloid differentiation factor 88) [21]. TLR signaling activates nuclear factor $(\mathrm{NF})-\kappa \mathrm{B}$ and mitogen-activated
Box 1. Conservation of flagellin detection systems in plants and mammals

In a similar manner to mammals, plants detect microbe-associated molecular patterns (MAMPs) from pathogenic microorganisms, known as general elicitors, via pattern-recognition receptors (PRRs) and then elicit a defense response. Similar to fungal cell-wall constituents (e.g. chitin, ergosterol and glucans), flagellin monomers of Pseudomonas syringae activate the production of reactive oxygen species, ion fluxes and ethylene in plants. A highly conserved linear motif of 22 residues close to the $\mathrm{N}$-terminus of flagellin ('QRLSTGSRINSAKDDAAGLQIA' for $P$. syringae) is recognized by FLS2, the flagellin-sensitive locus 2 product [57] (See Figures 1 and $3 b$ in the main text). FLS2 is a membranespanning protein composed of a leucine-rich repeat (LRR) domain and an intracellular signaling module, which functions as a serinethreonine kinase. Stimulation of FLS2 triggers rapid protein phosphorylation, the activation of mitogen-activated protein kinase (MAPK) cascade and the induction of defense-related genes. Because LRRs are not homologous in FLS2 and Toll-like receptor (TLR) 5 and flagellin-detected motifs are different, the LRR structure has probably been selected independently in plants and mammals to serve as a MAMP detection domain for flagellin of harmful flagellated bacteria.

protein kinase (MAPK) pathways that in turn modulate transcription of genes encoding immune mediators [21].

Flagellins exhibit some features of MAMPs, including sequence conservation and wide distribution among bacteria (Box 1). In mammals, TLR5 is involved in the detection of flagellin [22] (Figures 1 and 3b). Differences between human and mouse TLR5 sequences mediate the species-specific recognition of flagellins [23]. MyD88 is an essential adaptor molecule for TLR5 because responses to flagellin are totally abolished in MyD88-knockout animals $[22,24]$. TLR5 stimulates transcription of proinflammatory genes that are dependent on NF- $\mathrm{KB}$ and MAPKs, such as p38, JNK (c-Jun N-terminal kinase 1) and ERK1-2 (extracellular signal-regulated kinase 1 and 2) [22,25,26].

\section{Flagellin monomers mediate mucosal proinflammatory responses}

Low concentrations of flagellin [Effective dose $50\left(\mathrm{ED}_{50}\right)$ $\sim 10-50 \times 10^{-12} \mathrm{M}$ ] trigger proinflammatory signaling in sentinel cells $[27,28]$. Monocytes and macrophages produce proinflammatory cytokines [tumor necrosis factor (TNF)- $\alpha$ and interleukin (IL)-6] and/or nitric oxide [27,29]. Systemic injection of flagellin induces similar effects in mice $[22,30]$. In epithelial cells, the flagellins from S. enterica serovars Typhimurium, Dublin and Enteritidis, enteropathogenic $E$. coli and $P$. aeruginosa stimulate the polarized secretion of IL-8 (also named CXCL8), the chemokine essential for recruitment of neutrophils and macrophages at sites of injury [25,28,30-33]. Upon flagellin stimulation, epithelial cells also upregulate production of inducible nitric oxide synthase (iNOS) and nitric oxide, matrilysin [matrix metalloproteinase (MMP)-7], human $\beta$-defensin 2 (h- $\beta$ D2), and chemokines, such as CXCL2 [macrophage inflammatory protein (MIP)-2 $\alpha$ ] $[26,30,32]$. In mucosal tissues, these factors participate in anti-microbial activity, in the recruitment of professional killer and antigen-presenting phagocytes, and in the production of inflammatory mediators that set up the platform for phagocyte activation. Although virulence 
(a)

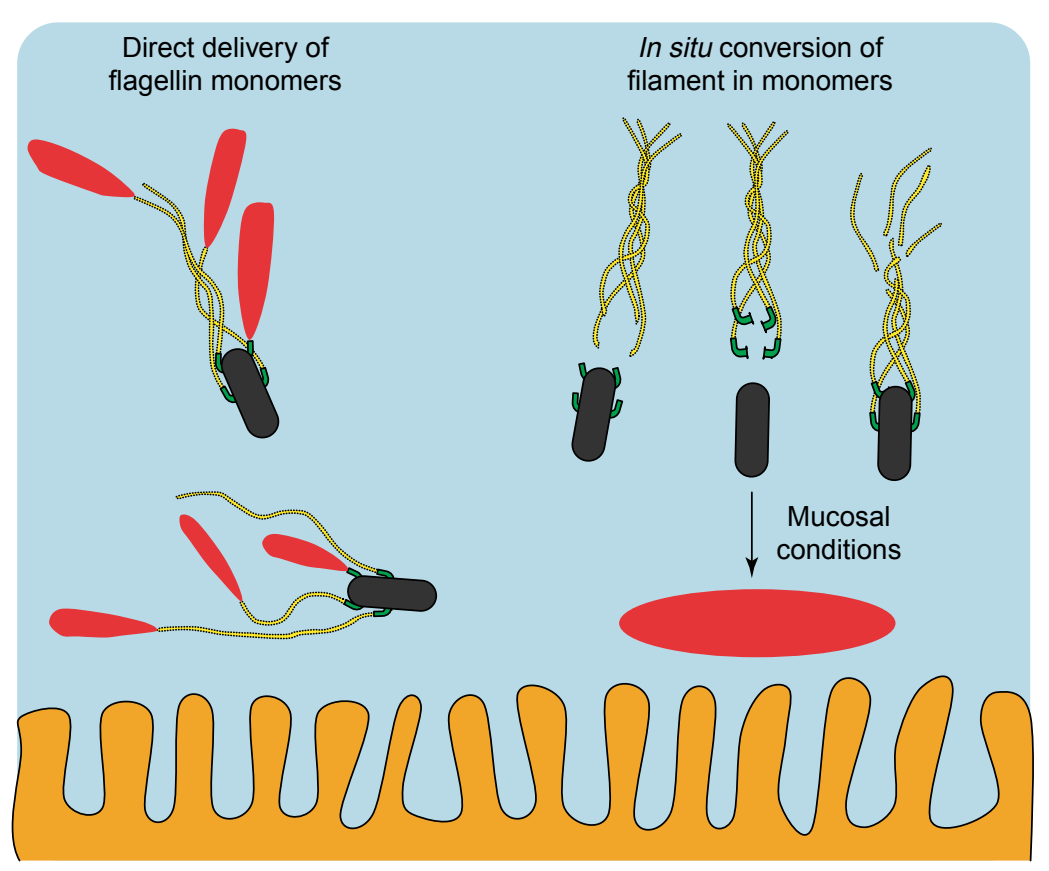

(b)

Mammals

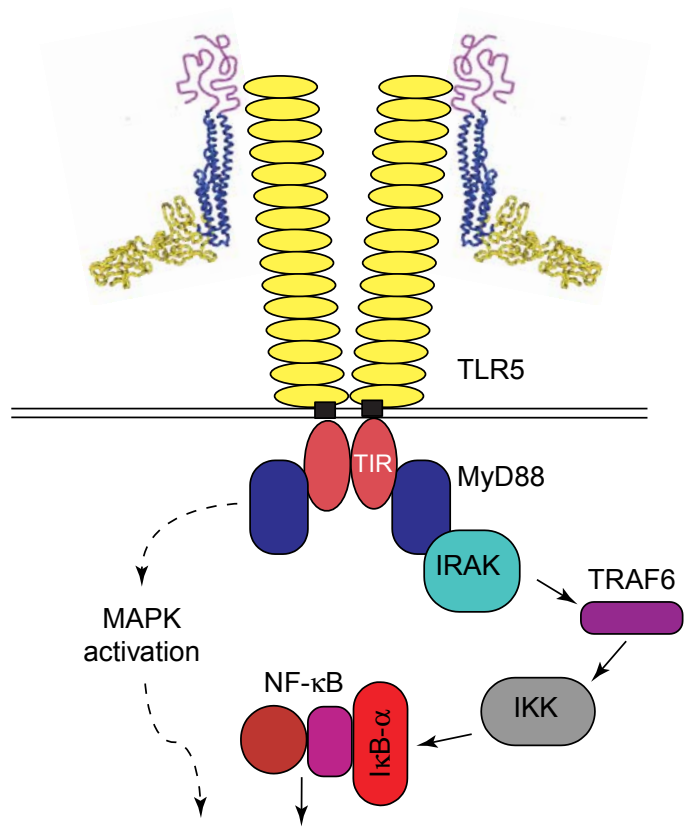

IL-8, CCL20, hßD-2, iNOS, MMP-7

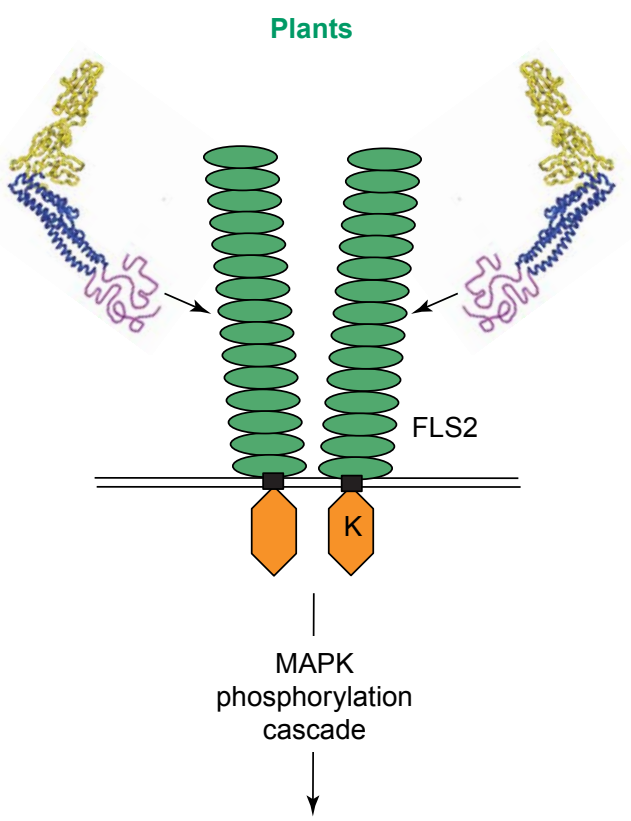

Release of hydrogen peroxide, superoxide, defense proteins

\section{$\overline{\text { TRENDS in Microbiology }}$}

Figure 3. Mucosal pro-inflammatory responses to flagellin monomers. (a) Models of flagellin monomer delivery in mucosal tissue. Some bacterial species secrete flagellin monomers (red) in culture owing to inefficient capping or flagellum break. This release might spontaneously occur in vivo or be controlled by host proteases and/or detergents shearing or uncapping flagella. When expression of flagellar components is already turned off, removal of flagella might occur, delivering intact filament into environment. A bacterial-regulated removal of flagellum might take place in the mucosa. Alternatively, host factors can cause flagellum break. The conversion of filament into flagellin monomers demands specific physico-chemical conditions that can be encountered in the mucosa. (b) Flagellin-specific pattern-recognition receptors and signal transduction pathways. Detection of flagellin monomer is performed by TLR5 in mammals and FLS2 in plants (Arabidopsis). Both transmembrane receptors are formed by extracellular leucine-rich repeats that detect distinct regions of flagellin monomers. TLR5 requires the universal TLR-specific adaptor molecule MyD88 that transfers the stimulus from TIR to downstream molecules of the signaling cascade, such as IRAK1, TRAF6, IKK and IKB- $\alpha$ molecules. Finally, the signaling results in activation of NF- $\mathrm{KB}$ and MAPK that turns on transcription of genes involved in innate and adaptive immunity. Shown here are some well-characterized factors that are upregulated upon flagellin stimulation of epithelial cells. In plants, flagellin activates the serine-threonine kinase (K) domain of FLS2, which induces a phosphorylation cascade of MAPKs and expression of plant defense mechanisms. Abbreviations: FLS2, flagellin-sensitive receptor; h- $\beta$ D2, human $\beta$-defensin 2; IKB- $\alpha$, inhibitor of NF-kappaB; IKK, I-kappa-B kinases; IL-8, interleukin 8; iNOS, nitric oxide synthase; IRAK1, interleukin-1 receptor-associated kinase; MAPK, mitogen-activated protein kinases; MMP-7, matrilysin; MyD88, myeloid differentiation factor 88; NF-אB, nuclear factor- $\mathrm{KB}$; TIR, Toll-interleukin 1 receptor domain; TLR5, Toll-like receptor 5; TRAF6, TNF receptor-associated factor 6. 
factors, such as invasins, were initially found to be required for the activation of proinflammatory responses by pathogenic bacteria in epithelial cells, recent studies showed that this response is specifically induced by flagellin $[25,26]$.

Epithelial cells of the gut and airways produce TLR5 in vitro and in vivo [34-36]. TLR5 expression was initially found on the basolateral surface of epithelial cells and dedicated to detection of invasive bacteria (i.e. S. enterica serovars) [35]. This distribution was also confirmed in vivo [30]. However, flagellin also activates apical signaling in epithelial cells, indicating that TLR5 is present in the luminal compartment $[25,32]$. Therefore, TLR5 is able to detect both extracellular-luminal and invasive-flagellated pathogenic bacteria.

\section{Relationship between flagellin structure and immunostimulatory activity}

The hypervariable domain of flagellins from pathogenic E. coli and Salmonella species is not required for TLR5 signaling [23,37]. This is consistent with stimulatory activity of Listeria monocytogenes flagellin, which lacks this variable region [22]. Monomers of flagellin induce TLR5 signaling, whereas filamentous flagella do not [23]. Indeed, the signaling moiety of flagellin is hidden in the flagellum but becomes accessible in the monomer (Figures 1 and $3 \mathrm{~b}$ ). Most mutations in the conserved D1 domain (residues 44-129 and 406-454 of $S$. typhimurium) reduce recognition of flagellin by TLR5 and have a profound effect on motility [23,37]. The hydrophobic motif $88-97$ in S. typhimurium, which is conserved in 377 flagellin sequences of various bacterial species, was proposed to interact with TLR5 [38]. TLR5 activation is independent of any post-translational modification of flagellin [23,39]. Interestingly, mechanisms developed by bacteria to escape TLR5 detection can depend on flagellin sequence (Box 2). TLR5 detects a specific conformation of flagellin domain $\mathrm{D} 1$, which is required for flagellum formation and function and is exposed only in monomeric form.

TLR5 is unique because the detected pattern is a protein. TLR5-flagellin complexes can be isolated suggesting direct protein-protein interactions $[23,40]$. Both LRR regions (1-407 and 386-636) of human TLR5 mediate binding of flagellin in contrast to the region defined by positions 1-386 [40]. Thus, Mizel et al. [40] proposed that the peptide 386-407 on TLR5 is a major flagellin-binding site. A putative flagellin-binding site (positions 552-561) in human TLR5 has also been proposed from bioinformatics analysis [38]. However, the two sites do not correlate with any function in signaling [35,40]. Remarkably, a natural truncated form of TLR5 (1-392) acts as a dominantnegative mutant on wild-type TLR5 [41]. In conclusion, further investigations are needed to decipher the molecular interactions of TLR5 with flagellin.

Another striking finding about TLR5 involves the recognition of a monomer ligand. The simplest hypothesis is that detection of one monomer by a single receptor results in signal transduction. Such interaction has been observed with recombinant LRR regions of TLR5 and flagellin [40]. However, the signaling function of TLR5 requires dimerization [22]. We can therefore speculate
Box 2. Bacterial strategies to escape flagellin-specific host immune responses

Activation of Toll-like receptors (TLRs) causes immune responses that are deleterious for any microorganisms. Although some flagellated pathogenic bacteria cope with defenses induced by TLR5 flagellin, others (pathogenic as well as non-pathogenic bacteria) use various strategies to prevent or hijack this activation [20]. Flagellated bacteria can produce flagellin that lack proinflammatory properties. The flagellins FlaA (Genbank accession number NP-207936) and FlaB (Genbank accession number NP-206915) from Helicobacter pylori preserve motility properties but not stimulatory activity for human TLR5 [58]. Interestingly, the primary sequences of the signaling D1 domain from Helicobacter flagellins are poorly homologous to TLR5-stimulatory flagellins, especially to the region 88-97 of Salmonella typhimurium flagellin [38]. Control can also operate at the level of flagellin expression. Bacteria that are flagellated when cultivated in vitro might not express flagellin in the host environment or only in a defined sequential virulence process. Alternatively, flagellin can be tightly enclosed in a highly stable flagellum or covered by a membranous sheath to prevent any release of monomers into the surrounding environment, such as in Vibrio cholerae and H. pylori. Finally, factors produced by bacteria can downregulate the pro-inflammatory signalling in response to their own signals or to MAMPS (microbeassociated molecular patterns), including flagellin. In order to prevent nuclear activation of nuclear factor (NF)- $\kappa B$-dependent genes, avirulent Salmonella pullorum blocks the degradation of I $\kappa \mathrm{B}-\alpha$, the cytosolic inhibitor of NF- $\kappa \mathrm{B}$, and commensal Bacteroides thetaiotamicron stimulates the nucleus-cytosol shuttling of RelA (a subunit of NF-KB) $[59,60]$. Nevertheless, the in vivo contribution of these various mechanisms remains to be established.

that TLR5 homodimers bind either two monomers in symmetric arrangement or one monomer in an asymmetric form. TLRs usually detect MAMPs formed of repetitive motifs, such as peptidoglycan. Such MAMPs aggregate many TLRs and thereby concentrate signaling machinery in focal complexes. By contrast, TLR5 combined to flagellin probably form individual signaling units. Such specificity might account for the effect of flagellin on adaptive immune responses [24].

Recent studies have proposed that TLR2, TLR4 and gangliosides, including asialo-GM1, cooperate with TLR5 as receptors for binding flagellin and/or signaling $[42,43]$. However, their contribution needs to be addressed to rule out the possible effects of other bacterial components in the preparations.

\section{Delivery of flagellin monomer in mucosa}

The mechanisms by which flagellin is released by bacteria during tissue colonisation are crucial for TLR5-mediated responses. Although flagellin is usually assembled in the flagellum, leakage and/or uncapping account for the in vitro secretion of flagellin [44] (Figure 3a). Flagellin might be released in mucosa by secretion as observed in vitro (Figure 3a), whereas delivery could be part of bacterial- and/or host-directed activities (Figure 3a). For example, Caulobacter crescentus ejects its flagellum when this organelle is no longer required for the bacterial life cycle [45]. Alternatively, flagella could be sheared from bacterial surfaces by host proteases or detergents, such as bile salts or surfactants. 
(a)

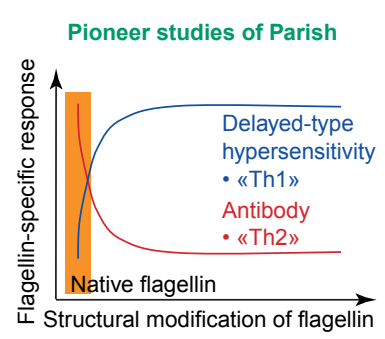

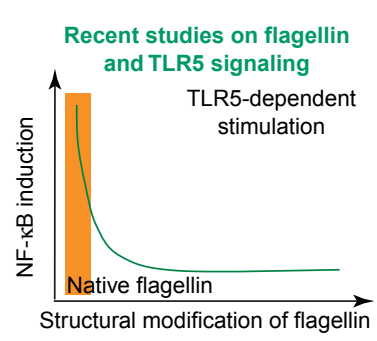

(b)

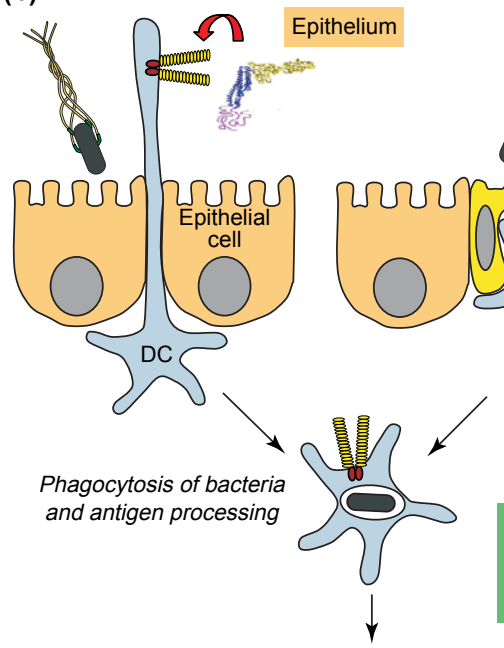

Mucosa-specific adaptive responses: Th2 and $\lg \mathrm{A}$
Skin
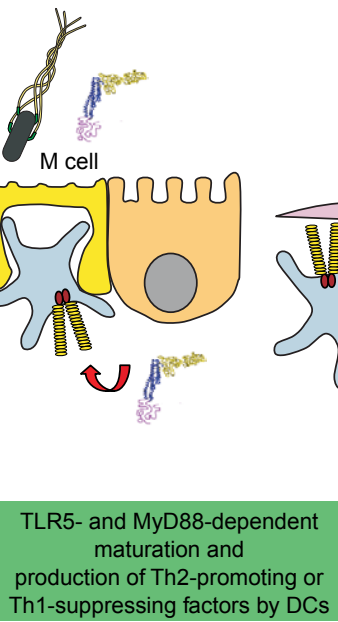

h1-suppressing factors by DCs

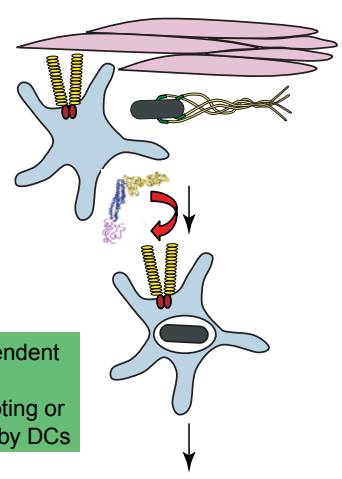

Systemic Th2-biased responses

(c)
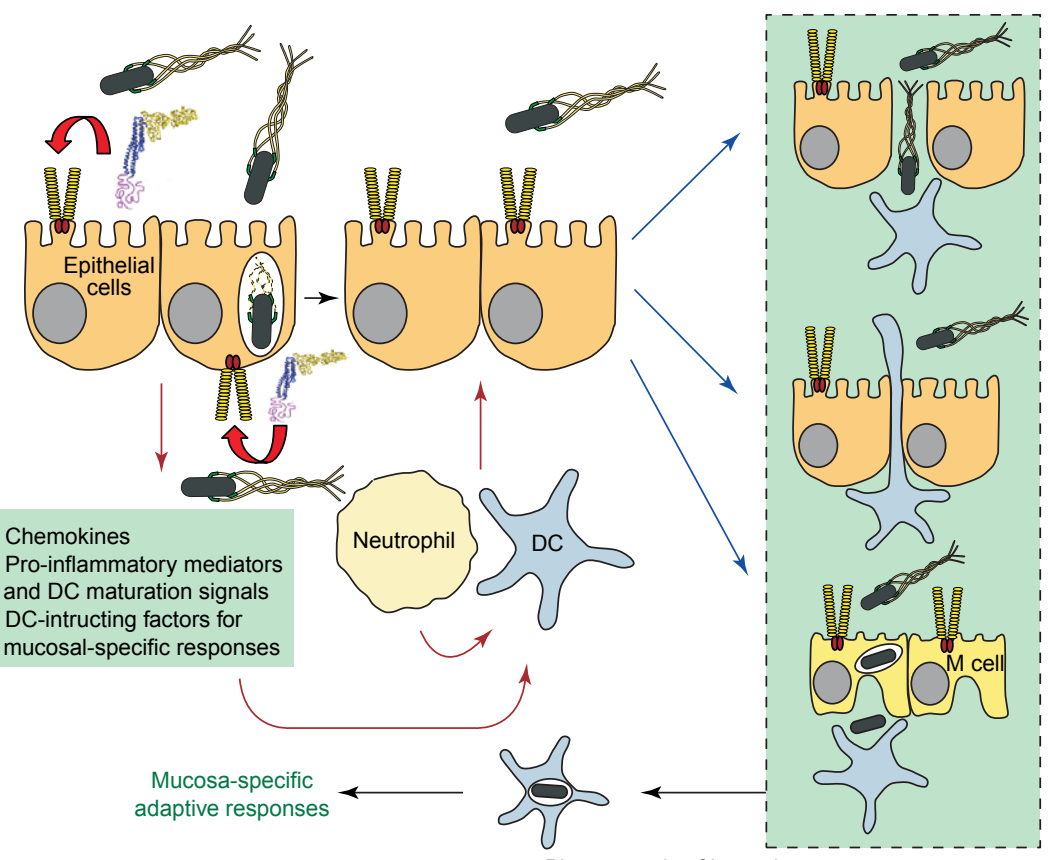

Phagocytosis of bacteria and antigen processing

$\overline{T R E N D S \text { in Microbiology }}$

Figure 4. Flagellin-specific regulation of adaptive immunity. (a) Correlation between Toll-like receptor (TLR) 5 stimulatory activity and flagellin structure. Left panel: the model of Parish considers Salmonella adelaide flagellin as a potent antigen that stimulates antibody responses in rats. By disturbing flagellin structure, antibody response is impaired but delayed-type hypersensitivity, which reflects the Th1 response, is stimulated. In these experiments, animals were primed with native (orange bar) or modified flagellin molecules that imprint the immune response, boosted with native flagellin and then assayed for antibody or delayed-type hypersensitivity. Right panel: TLR5 stimulatory activity is lost when mutations and/or insertions disturb the conserved domain of flagellin as presented schematically by the decrease in nuclear factor (NF)- $\kappa \mathrm{B}$ induction. (b) Flagellin-dependent activation of dendritic cells (DCs). Mucosal DCs that have membrane protrusions within the lumen are in direct contact with bacteria. $M$ cells (yellow) are specialized epithelial cells in intestinal Peyer's patches that transport and deliver luminal bacteria to subepithelial DCs. These DCs can be stimulated by flagellin that are released by bacteria. Flagellin induces DC maturation in a TLR5-, MyD88- and NF-kB-dependent manner. In this model, TLR5-signaling stimulates DCs to 


\section{Modulation of mucosal adaptive immune response by flagellin}

PRRs translate the pathogen-derived component into a specific 'identity card'. Thus, appropriate antibodies and T-cell responses are elicited to eradicate the pathogen. DCs are the key antigen-presenting cells that control the induction of adaptive immunity [46]. Immature DCs are resident sentinel cells in tissues that are specialized in antigen capture and can be activated by MAMPs. Upon activation, DCs migrate to draining lymphoid tissues where they present antigens to naïve $\mathrm{T}$ cells and provide signals for the development of appropriate $\mathrm{CD} 4^{+} \mathrm{T}$ helper (Th) responses [46]. CD4 ${ }^{+} \mathrm{T}$ cells committed to Th2 phenotype produce IL-4 and promote antibody responses, whereas Th1 cells promote a cell-mediated mechanism via interferon (IFN)- $\gamma$. Here, we will describe the studies that explored the TLR5-mediated effect of flagellin on adaptive immunity (Figure 4).

\section{TLR5: the paradigm of TLRs promoting Th2 and regulatory responses}

The pioneer studies conducted with flagellin that had been isolated from Salmonella adelaide showed that flagellin is a potent stimulator of antibody responses, which is a hallmark of Th2 responses (for a review see Ref. [47]) (Figure 4a). However, immune deviation towards delayedtype hypersensitivity, a hallmark of Th1 responses, was observed when flagellin structure was disturbed. Thus, this seminal work was instrumental in the elaboration of the paradigm of Th1-Th2 cells [47]. PRR-mediated DC activation can now provide a molecular clue. Recent works indicate that the primary signaling events, which initiate Th2-type responses, are mediated by TLR5. First, TLR5-stimulatory activity is lost when mutations perturb the conserved domain of flagellin [23,37] (Figure 4a). Although flagellin was initially reported to stimulate IFN- $\gamma$ production in mice [39], we recently established that flagellin promotes the development of Th2-biased (IL-4-producing $\mathrm{T}$ cells) and antibody responses [24] (Figure 4b). Flagellin induces maturation of TLR5-expressing DCs and the upregulation of co-stimulatory molecules and antigen-presenting capacity in a MyD88-dependent manner [24]. TLR5 signaling in DCs probably involves production of Th2-promoting or Th1-suppressing signals. In mouse or human DCs, flagellin does not enhance the production of IL-12 p70, a key cytokine in the development of Th1 cells, which could favor Th2 development [24,48]. An alternative mechanism for Th2-biased responses could be a moderate T-cellmediated suppression as a result of TLR5-stimulated DCs, a process involving an IL-6-dependent blockade of regulatory $\mathrm{T}$ cells $\left(\mathrm{CD} 4{ }^{+} \mathrm{CD} 25^{+}\right)$[49]. Finally, TLR5 signaling challenges the dogma that TLR activation promotes Th1 immunity, whereas Th2 responses are elicited by other PRRs [46].

Parish et al. [47] observed that immune deviation depends on flagellin dose, a phenomenon called high- and low-dose antibody tolerance, and this might also correlate with the TLR5-stimulatory activity. At low doses, there is no effective level of TLR5 agonist, whereas during stimulation with increasing doses of TLR5 agonist, first DCs and then regulatory CD4 ${ }^{+} \mathrm{T}$ cells might be directly activated. High doses of TLR ligands might stimulate the suppressive functions of regulatory T cells $\left(\mathrm{CD} 4{ }^{+} \mathrm{CD} 25^{+}\right)$ on $\mathrm{CD} 4^{+}$T-cell responses in a TLR-dependent manner [50]. Interestingly, regulatory T cells do express TLR5 [50].

\section{TLR5-dependent mucosal adaptive responses: $D C$ recruitment by epithelial cells}

Stimulation of mucosal immunity requires activation and antigen-uptake by lamina propria DCs [20,51]. In Peyer's patches, bacteria are transported by $\mathrm{M}$ cells from lumen to lamina propria where DCs are activated and promote imprinting of gut-specific T cells [51,52]. Although pathogens target such sites, they represent only a minor part of mucosa. DCs with membrane protrusions extending into the lumen might directly interact with bacteria throughout the mucosa [53]. The direct activation of DCs within mucosa by flagellin might participate in Th2 differentiation, which favors secretory antibody responses (Figure $4 \mathrm{~b}$ ).

Flagellin-mediated stimulation of epithelial cells is probably decisive for mucosal immune responses. Flagellin triggers the transient expression of the DC-specific chemokine CCL20 (also known as MIP-3 $\alpha$ or LARC) [32] (Figure 4c). Interestingly, ccl20 expression is constitutive in Peyer's patch epithelium and is associated with the sub-epithelial positioning of DCs producing CCR6, the CCL20-specific receptor [54]. CCR6-deficient mice have impaired mucosal adaptive immune responses [54]. Flagellin-treated epithelial cells attract immature DC in a CCL20-dependent manner [32]. At the same time, flagellin-stimulated epithelial cells secrete IL-8 and recruit neutrophils, providing appropriate proinflammatory signals for DC maturation [28]. ccl20 transcription is restricted to mucosal epithelium by mechanisms involving the epithelium-specific transcriptional factor ESE-1 and $\mathrm{NF}-\kappa \mathrm{B}$ [54-56]. Remarkably, lymphotoxin- $\beta$ activates NF-кB2 (RelB-p52) and sustained ccl20 expression, which might account for constitutive CCL20 production in Peyer's patch epithelium. By contrast, TLR5 causes NF- $\kappa$ B1 (p50-p65) activation and a short-lived expression of CCL20 in epithelial cells $[55,56]$. It is tempting to

shape Th2-biased immunity via production of Th2-promoting or Th1-suppressing factors. Such a mechanism, in combination with the epithelial environment, imprints DCs

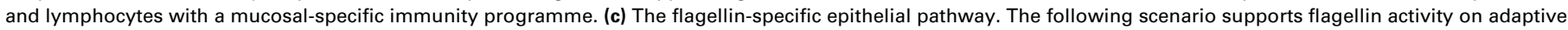

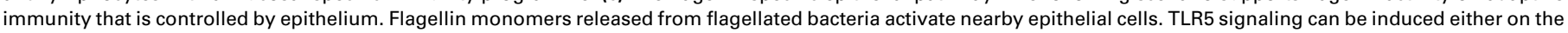

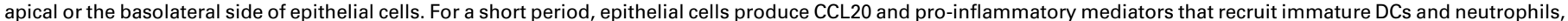

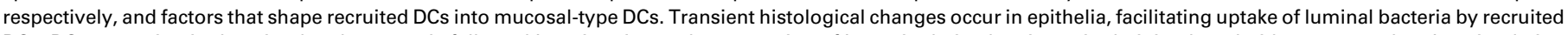

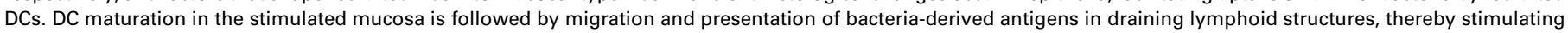

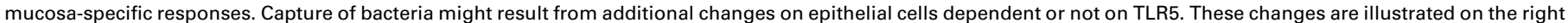

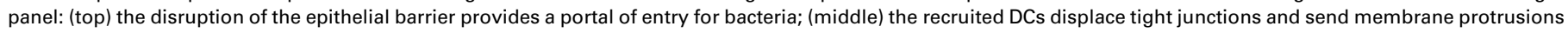
into the lumen; and (bottom) the epithelial cells become transiently capable to transport particles, such as the $\mathrm{M}$ cells of Peyer's patches. 
expand this analogy to include TLR-mediated epithelial 'education' of DCs to imprint the mucosal responses as described for Peyer's patch DCs (Figure 4c) [52].

The characterization of processes occurring after TLR activation in mucosa will be essential to decipher how adaptive immune system handle antigens and to develop new strategies for mucosal vaccination.

\section{Concluding remarks}

The widespread distribution of flagellin in bacteria and its use during pathogenesis result in elaborated mechanisms for recognition by eukaryotes. Now, the crystal structure of flagellar filament and flagellin provide outstanding information to investigate the contribution of the various domains of the molecule to the pathogenic and immunological processes. Combining this approach with genetically engineered animals, including mice conditionally deficient for TLR5, will help to decipher the role of mucosal and especially epithelial TLR5 in the host protection against flagellated bacteria. Recent findings emphasize the association of particular TLR5 polymorphisms in humans with the incidence of $L$. pneumophila infection [41], thereby providing new opportunities to control the human mucosal innate-adaptive immune systems.

\section{Acknowledgements}

We thank Dr. Kelly Hughes and Pr. Christopher R. Parish for outstanding suggestions. This work was supported by INSERM (programme AVENIR R02344ES), the Région Nord Pas de Calais (ARCir émergence), and the Fondation pour la Recherche Médicale.

\section{References}

1 Macnab, R.M. (2003) How bacteria assemble flagella. Annu. Rev. Microbiol. 57, 77-100

2 Yonekura, K. et al. (2003) Complete atomic model of the bacterial flagellar filament by electron cryomicroscopy. Nature 424, 643-650

3 Winstanley, C. and Morgan, J.A. (1997) The bacterial flagellin gene as a biomarker for detection, population genetics and epidemiological analysis. Microbiology 143, 3071-3084

4 Vonderviszt, F. et al. (1989) Terminal regions of flagellin are disordered in solution. J. Mol. Biol. 209, 127-133

5 Ottemann, K.M. and Miller, J.F. (1997) Roles for motility in bacterialhost interactions. Mol. Microbiol. 24, 1109-1117

6 Hughes, E.A. and Galan, J.E. (2002) Immune response to Salmonella: location, location, location? Immunity 16, 325-328

7 Krukonis, E.S. and DiRita, V.J. (2003) From motility to virulence: Sensing and responding to environmental signals in Vibrio cholerae. Curr. Opin. Microbiol. 6, 186-190

8 Drake, D. and Montie, T.C. (1988) Flagella, motility and invasive virulence of Pseudomonas aeruginosa. J. Gen. Microbiol. 134, 43-52

9 Ottemann, K.M. and Lowenthal, A.C. (2002) Helicobacter pylori uses motility for initial colonization and to attain robust infection. Infect. Immun. 70, 1984-1990

10 Marchetti, M. et al. (2004) Interaction of pathogenic bacteria with rabbit appendix M cells: bacterial motility is a key feature in vivo. Microbes Infect. 6, 521-528

11 Tasteyre, A. et al. (2001) Role of FliC and FliD flagellar proteins of Clostridium difficile in adherence and gut colonization. Infect. Immun. 69, 7937-7940

12 Lillehoj, E.P. et al. (2002) Identification of Pseudomonas aeruginosa flagellin as an adhesin for Muc1 mucin. Am. J. Physiol. Lung Cell. Mol. Physiol. 282, L751-L756

13 Giron, J.A. et al. (2002) The flagella of enteropathogenic Escherichia coli mediate adherence to epithelial cells. Mol. Microbiol. 44, 361-379

14 Young, G.M. et al. (2000) Motility is required to initiate host cell invasion by Yersinia enterocolitica. Infect. Immun. 68, 4323-4326

15 Galan, J.E. (2001) Salmonella interactions with host cells: type III secretion at work. Annu. Rev. Cell Dev. Biol. 17, 53-86
16 Young, G.M. et al. (1999) A new pathway for the secretion of virulence factors by bacteria: the flagellar export apparatus functions as a protein-secretion system. Proc. Natl. Acad. Sci. U. S. A. 96, 6456-6461

17 Chilcott, G.S. and Hughes, K.T. (2000) Coupling of flagellar gene expression to flagellar assembly in Salmonella enterica serovar typhimurium and Escherichia coli. Microbiol. Mol. Biol. Rev. 64, 694-708

18 Goodier, R.I. and Ahmer, B.M. (2001) SirA orthologs affect both motility and virulence. J. Bacteriol. 183, 2249-2258

19 Akerley, B.J. et al. (1992) The bvgAS locus negatively controls motility and synthesis of flagella in Bordetella bronchiseptica. J. Bacteriol. $174,980-990$

20 Niedergang, F. et al. (2004) Dendritic cells: the host Achille's heel for mucosal pathogens? Trends Microbiol. 12, 79-88

21 Akira, S. and Takeda, K. (2004) Toll-like receptor signalling. Nat. Rev. Immunol. 4, 499-511

22 Hayashi, F. et al. (2001) The innate immune response to bacterial flagellin is mediated by Toll-like receptor 5 . Nature 410, 1099-1103

23 Smith, K.D. et al. (2003) Toll-like receptor 5 recognizes a conserved site on flagellin required for protofilament formation and bacterial motility. Nat. Immunol. 4, 1247-1253

24 Didierlaurent, A. et al. (2004) Flagellin Promotes Myeloid Differentiation Factor 88-Dependent Development of Th2-Type Response. J. Immunol. 172, 6922-6930

25 Berin, M.C. et al. (2002) Role of EHEC O157:H7 virulence factors in the activation of intestinal epithelial cell NF-kappaB and MAP kinase pathways and the upregulated expression of interleukin 8. Cell. Microbiol. 4, 635-648

26 Zeng, H. et al. (2003) Flagellin is the major proinflammatory determinant of enteropathogenic Salmonella. J. Immunol. 171, 3668-3674

27 McDermott, P.F. et al. (2000) High-affinity interaction between gramnegative flagellin and a cell surface polypeptide results in human monocyte activation. Infect. Immun. 68, 5525-5529

28 Gewirtz, A.T. et al. (2001) Salmonella typhimurium translocates flagellin across intestinal epithelia, inducing a proinflammatory response. J. Clin. Invest. 107, 99-109

29 Moors, M.A. et al. (2001) Activation of interleukin-1 receptorassociated kinase by gram-negative flagellin. Infect. Immun. 69, 4424-4429

30 Eaves-Pyles, T. et al. (2001) Flagellin, a novel mediator of Salmonellainduced epithelial activation and systemic inflammation: I kappa $\mathrm{B}$ alpha degradation, induction of nitric oxide synthase, induction of proinflammatory mediators, and cardiovascular dysfunction. J. Immunol. 166, 1248-1260

31 Steiner, T.S. et al. (2000) Enteroaggregative Escherichia coli expresses a novel flagellin that causes IL-8 release from intestinal epithelial cells. J. Clin. Invest. 105, 1769-1777

32 Sierro, F. et al. (2001) Flagellin stimulation of intestinal epithelial cells triggers CCL20-mediated migration of dendritic cells. Proc. Natl. Acad. Sci. U. S. A. 98, 13722-13727

33 Hybiske, K. et al. (2004) Cystic fibrosis airway epithelial cell polarity and bacterial flagellin determine host response to Pseudomonas aeruginosa. Cell. Microbiol. 6, 49-63

34 Cario, E. and Podolsky, D.K. (2000) Differential alteration in intestinal epithelial cell expression of Toll-like receptor 3 (TLR3) and TLR4 in inflammatory bowel disease. Infect. Immun. 68, 7010-7017

35 Gewirtz, A.T. et al. (2001) Bacterial flagellin activates basolaterally expressed TLR5 to induce epithelial proinflammatory gene expression. J. Immunol. 167, 1882-1885

36 Muir, A. et al. (2004) Toll-like receptors in normal and cystic fibrosis airway epithelial cells. Am. J. Respir. Cell Mol. Biol. 30, 777-783

37 Eaves-Pyles, T.D. et al. (2001) Salmonella flagellin-dependent proinflammatory responses are localized to the conserved amino and carboxyl regions of the protein. J. Immunol. 167, 7009-7016

38 Jacchieri, S.G. et al. (2003) Structural study of binding of flagellin by Toll-like receptor 5. J. Bacteriol. 185, 4243-4247

39 McSorley, S.J. et al. (2002) Bacterial flagellin is an effective adjuvant for CD4(+) T cells in vivo. J. Immunol. 169, 3914-3919

40 Mizel, S.B. et al. (2003) Identification of a sequence in human toll-like receptor 5 required for the binding of Gram-negative flagellin. J. Biol. Chem. 278, 23624-23629 
41 Hawn, T.R. et al. (2003) A Common Dominant TLR5 Stop Codon Polymorphism Abolishes Flagellin Signaling and Is Associated with Susceptibility to Legionnaires' Disease. J. Exp. Med. 198, 1563-1572

42 Adamo, R. et al. (2004) Pseudomonas aeruginosa flagella activate airway epithelial cells through asialoGM1 and Toll-like receptor 2 as well as Toll-like receptor 5. Am. J. Respir. Cell Mol. Biol. 30, 627-634

43 Mizel, S.B. et al. (2003) Induction of macrophage nitric oxide production by gram-negative flagellin involves signaling via heteromeric toll-like receptor 5/toll-like receptor 4 complexes. J. Immunol. $170,6217-6223$

44 Komoriya, K. et al. (1999) Flagellar proteins and type III-exported virulence factors are the predominant proteins secreted into the culture media of Salmonella typhimurium. Mol. Microbiol. 34, 767-779

45 Jenal, U. and Stephens, C. (2002) The Caulobacter cell cycle: timing, spatial organization and checkpoints. Curr. Opin. Microbiol. 5, $558-563$

46 Kapsenberg, M.L. (2003) Dendritic-cell control of pathogen-driven T-cell polarization. Nat. Rev. Immunol. 3, 984-993

47 Parish, C.R. (1996) Immune deviation: a historical perspective. Immunol. Cell Biol. 74, 449-456

48 Means, T.K. et al. (2003) The Toll-like receptor 5 stimulus bacterial flagellin induces maturation and chemokine production in human dendritic cells. J. Immunol. 170, 5165-5175

49 Pasare, C. and Medzhitov, R. (2003) Toll pathway-dependent blockade of CD4+CD25 + T cell-mediated suppression by dendritic cells. Science 299, 1033-1036

50 Caramalho, I. et al. (2003) Regulatory T Cells Selectively Express Toll-like Receptors and Are Activated by Lipopolysaccharide. J. Exp. Med. 197, 403-411
51 Macpherson, A.J. and Uhr, T. (2004) Induction of protective IgA by intestinal dendritic cells carrying commensal bacteria. Science 303, 1662-1665

52 Mora, J.R. et al. (2003) Selective imprinting of gut-homing T cells by Peyer's patch dendritic cells. Nature 424, 88-93

53 Rescigno, M. et al. (2001) Dendritic cells express tight junction proteins and penetrate gut epithelial monolayers to sample bacteria. Nat. Immunol. 2, 361-367

54 Cook, D.N. et al. (2000) CCR6 mediates dendritic cell localization, lymphocyte homeostasis, and immune responses in mucosal tissue. Immunity 12, 495-503

55 Kwon, J.H. et al. (2003) ESE-1, an Enterocyte-specific Ets Transcription Factor, Regulates MIP-3alpha Gene Expression in Caco-2 Human Colonic Epithelial Cells. J. Biol. Chem. 278, 875-884

56 Rumbo, M. et al. (2004) Lymphotoxin beta receptor signaling induces the chemokine CCL20 in intestinal epithelium. Gastroenterology 127, 213-223

57 Gomez-Gomez, L. and Boller, T. (2002) Flagellin perception: a paradigm for innate immunity. Trends Plant Sci. 7, 251-256

58 Lee, S.K. et al. (2003) Helicobacter pylori flagellins have very low intrinsic activity to stimulate human gastric epithelial cells via TLR5. Microbes Infect. 5, 1345-1356

59 Neish, A.S. et al. (2000) Prokaryotic regulation of epithelial responses by inhibition of I kappa B-alpha ubiquitination. Science 289, 1560-1563

60 Kelly, D. et al. (2004) Commensal anaerobic gut bacteria attenuate inflammation by regulating nuclear-cytoplasmic shuttling of PPAR-gamma and RelA. Nat. Immunol. 5, 104-112

\section{Have you contributed to an Elsevier publication?}

\section{Did you know that you are entitled to a $\mathbf{3 0} \%$ discount on books?}

A $30 \%$ discount is available to ALL Elsevier book and journal contributors when ordering books or stand-alone CD-ROMs directly from us.

To take advantage of your discount:

1. Choose your book(s) from www.elsevier.com or www.books.elsevier.com

2. Place your order

Americas:

TEL: +18007824927 for US customers

TEL: +1800 4603110 for Canada, South \& Central America customers

FAX: +1314 4534898

E-MAIL: author.contributor@elsevier.com

All other countries:

TEL: +44 1865474010

FAX: +441865 474011

E-MAIL: directorders@elsevier.com

You'll need to provide the name of the Elsevier book or journal to which you have contributed. Shipping is FREE on pre-paid orders within the US, Canada, and the UK.

If you are faxing your order, please enclose a copy of this page.

3. Make your payment

This discount is only available on prepaid orders. Please note that this offer does not apply to multi-volume reference works or Elsevier Health Sciences products. 